\title{
Asociación entre el nivel de competencias digitales docentes y variables sociodemográficas en docentes de educación media superior
}

\author{
Abel Olivas Martínez ${ }^{a}$, Dora Luz González-Bañales ${ }^{b}$ \\ ${ }^{a}$ Centro de Bachillerato Tecnológico Industrial y de Servicios 130, México, abelobin@gmail.com \\ bInstituto Tecnológico de Durango, Tecnológico Nacional de México, doraglez@itdurango.edu.mx
}

\begin{abstract}
Resumen
La incorporación de diversas herramientas tecnológicas en el sector educativo en todos los niveles, ha planteado en años recientes nuevos retos para los docentes, dentro de estos las denominadas Competencias Digitales Docentes (CDD) que implican que el profesor sea capaz de utilizar la tecnología para mejorar y transformar las prácticas educativas, así como pensar críticamente acerca del por qué, cómo y cuándo aprender nuevos aspectos relacionados con la tecnología y el proceso de enseñanzaaprendizaje. Los resultados que se presentan en este trabajo analizan la asociación entre la autopercepción del nivel de CDD (niveles: principiante, medio, experto y transformador) con variables sociodemográficas (género, edad, años de experiencia docente y grado de estudios). El encuadre metodológico es cuantitativo, no experimental y transversal. El instrumento utilizado se aplicó a 197 docentes de educación media superior en una región del Norte de México, y se diseñó a partir de la adaptación de estándares de Tecnologías de Información y Comunicación (TIC) para profesores del International Society for Technology in Education (ISTE) y del modelo de Krumsvik, así como algunos atributos referentes al uso de las TIC que marca el acuerdo 447 de la Reforma Integral de Educación Media Superior (RIEMS) de México. Se utilizaron las pruebas de Chi-Cuadrado y U de Mann-Whitney la primera para analizar la asociación entre variables edad, años de experiencia docente y grado de estudios, y la segunda para comparar diferencias a razón de la variable género. Los resultados estadísticos sugieren que existe una asociación entre las CDD con las variables edad y experiencia docente (medida en años de servicio) $y$ diferencias en el nivel autopercibido de CDD con la variable género.
\end{abstract}

Palabras clave: Competencias Digitales Docentes, Alfabetización Tecnológica, Variables sociodemográficas. 


\section{Introducción}

La incorporación de las TIC en educación plantea nuevos retos para los docentes, quienes deben de contar con una diversidad de competencias requeridas en el contexto de las exigencias de la nueva sociedad del conocimiento, dentro de ellas las denominadas competencias digitales $(\mathrm{CD})$.

Existen diversas acepciones sobre $\mathrm{CD}$ y algunas de ellas han sido abordadas por instituciones internacionales tales como UNESCO, Parlamento Europeo, Ministerios de educación en varios países. En la publicación de la Recomendación del Parlamento Europeo y del Consejo sobre las competencias clave para el aprendizaje permanente (DO L394, 2006) se reconoce a la CD como una de las ocho competencias clave dentro del "Programa de Aprendizaje Permanente" de la Unión Europea, siendo en dicho documento donde se define por primera vez la competencia digital como:

La competencia digital entraña el uso seguro y crítico de las tecnologías de la sociedad de la información (TSI) para el trabajo, el ocio y la comunicación. Se sustenta en las competencias básicas en materia de TIC: el uso de ordenadores para obtener, evaluar, almacenar, producir, presentar e intercambiar información, y comunicarse y participar en redes de colaboración a través de Internet (DO L394, 2006, como se citó en Pérez Escoda, 2015, p. 340).

Diversos autores basan la definición de CD en el desarrollo de habilidades, actitudes, aptitudes y en la capacidad de utilizar las TIC, de manera eficaz y eficiente (DO L394, 2006; Esteve-Mon, 2015; Krumsvik, 2014). Sin embargo, las habilidades son solo parte de los dominios de aprendizaje que se incluyen en la propia CD y la capacidad de utilizar herramientas o aplicaciones específicas, ya que solo es una de las diversas áreas de competencia que los usuarios deben desarrollar para funcionar en un entorno digital (Ferrari, 2012).

El hacer un uso efectivo de las TIC en el ámbito educativo, podría sugerir que los docentes aplicarían dichas CD para implementar nuevas y mejores estrategias en el proceso de enseñanza-aprendizaje; la presente investigación se centra en analizar la asociación entre CDD (niveles: principiante, medio, experto y transformador) con variables sociodemográficas (género, edad, años de experiencia docente y grado de estudios) en profesores de nivel medio superior, concretamente de los planteles de la Unidad de Educación Media Superior Tecnológica Industrial y de Servicios (UEMSTIS), del municipio de Durango, Estado de Durango, México.

La investigación de la cual se desprende esta ponencia parte del supuesto de que si los docentes utilizan las TIC antes, durante y después de su cátedra, sugeriría que se debería presentar un mejor desempeño en la manera o forma de enseñar e incluso su nivel de 
innovación utilizando TIC, sin embargo “...con frecuencia, los profesores consideran que es urgente hacer cosas, actuar con un sentido técnico o desarrollar actividades innovadoras, sin detenerse a pensar en por qué actúan así y cómo influyen sus decisiones en los resultados que obtienen" (Oviedo et al., 2010, p. 32). Con base en lo anterior, surge al mismo tiempo el interés en conocer si una serie de variables sociodemográficas, dentro de ellas el género y la edad, se asocian con el hecho de hacer un uso más o menos efectivo de las TIC, en los procesos de enseñanza-aprendizaje; es por eso, que la problemática de esta investigación se desprende de la posibilidad de identificar e indagar desde la autopercepción de los docentes su nivel de competencia digital y determinar la existencia de una asociación con variables sociodemográficas, siendo la pregunta de investigación: ¿Existe asociación entre el nivel de CDD y las variables sociodemográficas edad, años de experiencia docente y grado de estudios? ¿Existe diferencia entre el nivel de CDD y la variable género?

\section{Marco conceptual}

\section{Competencias Digitales Docentes}

Las definiciones del concepto "competencia" son múltiples, siendo una de ellas aquella que dice: "se trata de un concepto complejo, controvertido, con múltiples matices y a menudo polisémico, según el ámbito en el que se utiliza, y más aún si tenemos en cuenta las múltiples variables y denominaciones de esta expresión en otras lenguas" (Esteve-Mon, 2015, p. 45). En este sentido varios autores coinciden en la combinación dinámica de atributos: en relación a conocimientos, capacidades, habilidades, actitudes y responsabilidades, para responder a las demandas a las que hace frente una persona con un determinado nivel de eficiencia y eficacia (Gimeno Sacristán, 2008; OCDE, 2005; Perrenoud, 2010).

En lo que respecta a la $\mathrm{CD}$, esta es una de las competencias básicas para el siglo XXI, y entendida como las actitudes, aptitudes, destrezas, habilidades y conocimientos básicos en el uso de las TIC para hacer frente a los nuevos retos de la sociedad, por lo tanto, es básica para cualquier ciudadano, siendo entonces, muy necesaria para la actuación del docente (Hernández, Acevedo, Martínez, \& Cruz, 2014).

Sin embargo, la realidad es que resulta ser incómodo o complicado para algunos profesores, quienes en ocasiones no cuentan con los conocimientos y habilidades para ello; por ende, se les complica la implementación de las TIC en el aula o en el último de los casos pueden negarse a hacerlo. Para entender el papel del docente y su responsabilidad de lo que se ha comentado previamente es necesario conocer algunas definiciones sobre CDD, la cual se refiere a: 
las habilidades, actitudes y conocimientos requeridos por los educadores para apoyar el aprendizaje del alumno en un mundo digital rico, estos deben ser capaces de utilizar la tecnología para mejorar y transformar las prácticas de aula y enriquecer su propio desarrollo e identidad, así como pensar críticamente acerca del por qué, cómo y cuándo aprender nuevos aspectos relacionados con la tecnología y la enseñanza (Hall et al., 2014, como se citó en Esteve-Mon, 2015, p. 79).

Por otro lado Krumsvik (2009) define la alfabetización digital (sinónimo de competencia digital docente (Esteve-Mon, 2015)), como la capacidad del profesor para utilizar las TIC en un contexto profesional con un buen juicio pedagógico-didáctico y su conciencia de sus implicaciones en las estrategias de aprendizaje y en la formación digital de alumnos.

Asimismo, otros autores como Marquès (2008) señala que las CDD, son las mismas que requieren todos los ciudadanos, además de aquellas de carácter específico que se derivan de la aplicación de la tecnología en su labor profesional para mejorar los procesos de enseñanza, aprendizaje y gestión.

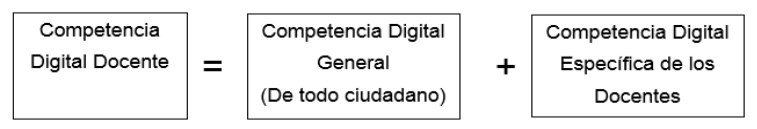

Figura 1. Competencia Digital Docente

Fuente: elaboración propia a partir de Marquès (Marquès, 2008)

La definición que se toma como base para esta investigación es la Krumsvik (2009), ya que se orienta a identificar el nivel conciencia y competencia digital docente a partir de su propia autopercepción.

\section{Metodología}

La presente investigación es de tipo cuantitativa, no experimental y transversal, teniendo como instrumento de recopilación de datos el cuestionario, mismo que fue aplicado en versión en línea (Google Formularios). El cuestionario se aplicó a 197 docentes de educación media superior en la ciudad de Durango, México (Norte de México).

Para el diseño y elaboración del instrumento, denominado Cuestionario de Autopercepción de la Competencia Digital para Docentes de Educación Media Superior (CACDDEMS), el cual consta de un total de 40 ítems, se realizó una adaptación al Cuestionario de Autopercepción de la Competencia Digital Docente (CACDD) propuesto por Esteve-Mon (2015), del cual se extrajeron 20 ítems, basados en los estándares de tecnología educativa para profesores (NETS-T) de ISTE (2008) y en las recomendaciones de Krumsvik (2009; 2014). Se incorporaron 12 ítems del Cuestionario a Profesores de Educación Primaria de Castilla y León (CPEPCL), desarrollado por Pérez Escoda (2015), basado en el TPACK 
("Technological Pedagogical Content Knowledge" (Koehler \& Mishra, 2008)). De manera complementaria se propusieron ocho ítems, tomando en consideración algunos atributos referentes al uso de las TIC que marca el acuerdo 447 en México (DOF, 2008).

En lo referente al cuestionario éste se organizó en dos secciones: una con variables sociodemográficas y otra con los ítems del CACDDEMS, estos últimos, a su vez se dividieron entre las cinco dimensiones del NETST-T de ISTE (2008), cada una de ellas con ocho ítems. En la Tabla 1 se presentan los principales ítems.

Tabla 1. Dimensiones y principales ítems del CACDDEMS

\begin{tabular}{|c|c|}
\hline Dimensión & Ítem \\
\hline \multirow{4}{*}{$\begin{array}{l}\text { I. Facilitar e } \\
\text { inspirar el } \\
\text { aprendizaje y la } \\
\text { creatividad los } \\
\text { estudiantes }\end{array}$} & $\begin{array}{l}\text { Adaptar formas adecuadas de utilización de las TIC para incrementar el pensamiento } \\
\text { creativo de mis alumnos. }\end{array}$ \\
\hline & $\begin{array}{l}\text { Utilizar las TIC con un programa o aplicación didáctica y estratégica en distintos ambientes } \\
\text { de aprendizaje. }\end{array}$ \\
\hline & Implementar actividades didácticas con TIC para mis alumnos, basadas en problemas reales. \\
\hline & $\begin{array}{l}\text { Elaborar recursos (presentaciones, videos, sonidos) con distintas herramientas (PowerPoint, } \\
\text { Prezi, Photoshop, Moviemaker, etc.) para facilitar el aprendizaje. }\end{array}$ \\
\hline \multirow{4}{*}{$\begin{array}{c}\text { II. Diseñar y } \\
\text { desarrollar } \\
\text { experiencias de } \\
\text { aprendizaje y } \\
\text { evaluaciones } \\
\text { propias de la era } \\
\text { digital }\end{array}$} & $\begin{array}{l}\text { Interactuar con herramientas TIC (por ejemplo: computadora, tableta, celular, cañón o } \\
\text { proyector, pizarrón digital) en actividades didácticas de un aula. }\end{array}$ \\
\hline & $\begin{array}{l}\text { Crear contenidos nuevos (textos, imágenes, gráficos, mapas mentales) con herramientas } \\
\text { digitales (Word, PowerPoint, Prezi, etc.). }\end{array}$ \\
\hline & Proponer actividades con TIC donde el alumno tenga un papel activo y de investigación. \\
\hline & $\begin{array}{l}\text { Realizar evaluaciones formativas y sumativas mediadas por las TIC para mejorar el } \\
\text { aprendizaje y la enseñanza. }\end{array}$ \\
\hline \multirow{4}{*}{$\begin{array}{l}\text { III. Modelar el } \\
\text { trabajo y el } \\
\text { aprendizaje } \\
\text { característicos } \\
\text { de la era digital }\end{array}$} & $\begin{array}{l}\text { Gestionar información digital (Buscar, seleccionar y almacenar) en: navegadores, } \\
\text { buscadores, dispositivos fijos (Disco duro), dispositivos portables (USB, Disco duro), } \\
\text { repositorios en la nube (Dropbox, Google Drive, etc.). }\end{array}$ \\
\hline & $\begin{array}{l}\text { Utilizar herramientas sociales y colaborativas (por ejemplo: blogs, foros, chats; Moodle, } \\
\text { Schoology, Chamilo, Classroom, etc.) para que mis alumnos trabajen y aprendan. }\end{array}$ \\
\hline & $\begin{array}{l}\text { Participar y comunicarme en entornos digitales con compañeros de trabajo y alumnos } \\
\text { (Twitter, Facebook, LinkedIn, Whatsapp, etc.). }\end{array}$ \\
\hline & $\begin{array}{l}\text { Tener nociones de informática (sistema operativo, instalación de programas (software) y } \\
\text { equipo (hardware), configuración de dispositivos, etc.). }\end{array}$ \\
\hline \multirow{4}{*}{$\begin{array}{l}\text { IV. Promover y } \\
\text { ejemplificar } \\
\text { ciudadanía } \\
\text { digital y } \\
\text { responsabilidad }\end{array}$} & $\begin{array}{l}\text { Evitar riesgos relacionados con la tecnología: exceso de tiempo expuesto a Internet, } \\
\text { adicciones, ciberacoso, etc. }\end{array}$ \\
\hline & $\begin{array}{l}\text { Aplicar prácticas éticas y legales de los recursos digitales, como derechos de la propiedad } \\
\text { intelectual y las licencias de uso en software e Internet. }\end{array}$ \\
\hline & $\begin{array}{l}\text { Proteger mi equipo con antivirus y conocer los sistemas de seguridad digitales (instalación } \\
\text { de contraseñas, etc.). }\end{array}$ \\
\hline & $\begin{array}{l}\text { Proteger mis datos personales y mi identidad digital, siendo consciente de la información } \\
\text { privada que subo a la red. }\end{array}$ \\
\hline \multirow{4}{*}{$\begin{array}{l}\text { V. Compromiso } \\
\text { con el } \\
\text { crecimiento } \\
\text { profesional y } \\
\text { con el liderazgo }\end{array}$} & Cumplir la propuesta de actualización en el uso de las TIC, al menos una vez al año. \\
\hline & $\begin{array}{l}\text { Localizar comunidades locales y nacionales de docentes para seguir aprendiendo sobre el } \\
\text { uso de las TIC en educación. }\end{array}$ \\
\hline & Diseñar estrategias utilizando las TIC para seguir aprendiendo como docente. \\
\hline & Revisar acerca de investigaciones educativas publicadas para el uso de las TIC. \\
\hline
\end{tabular}
Escoda (2015). 
Asociación entre el nivel de competencias digitales docentes y variables sociodemográficas en docentes de educación media superior

La medida de cada ítem fue con base en la afirmación "me siento capaz de..." formulada en escala tipo Likert (1. Nada Capaz al 5. Muy Capaz). El valor de Alpha de Cronbach fue de .984 .

Para obtener el nivel de CDD desde la autopercepción del docente, se generó una Variable General de las CDD a partir de la media de los resultados de cada una de las dimensiones a través de un baremo para determinar con ello el nivel de CDD, como se presenta en la Tabla 2 .

Tabla 2. Baremo para determinar el nivel de las CDD

\begin{tabular}{|c|c|c|}
\hline Nivel & Rango & Intervalo \\
\hline Principiante - Adopción & $1.00-1.99$ & 1.00 \\
\hline Medio - Adaptación & $2.00-3.49$ & 1.50 \\
\hline Experto - Apropiación & $3.50-4.49$ & 1.00 \\
\hline Transformador - Innovador & $4.50-5.00$ & 0.50 \\
\hline
\end{tabular}

Una de las razones por la cual la escala del baremo no es proporcional es porque en las pruebas que se hicieron en la fase de pilotaje del instrumento al analizar las frecuencias que se generaban, la mayoría de éstas se cargaban demasiado al nivel Transformador Innovador, por lo que los resultados se presentaban muy sesgados, por lo cual utilizando un decremento en los rangos a partir del segundo nivel, la percepción fue más apegada a la realidad del contexto.

\section{Resultados}

\subsection{Variables sociodemográficas}

En el estudio participaron 197 docentes de educación media superior ubicados en la ciudad de Durango, México. La edad promedio de los participantes fue de 41 años. Del total de los encuestados, 15\% (29) están dentro del rango de edad de 23 a 29 años; un 26\% (51) entre 30 a 36 años; de entre los 37 a 43 años un 21\% (41) y 32\% (62) entre 44 a 60 años; el 6\% (14) docentes con más de 60 años. En lo referente a la experiencia docente el 30.5\% (60) tienen de 1 a 5 años de experiencia docente (principiantes), 19\% (39) de 6 a 10 años de experiencia (experiencia básica); 22\% (44) de 11 a 20 años de experiencia (experiencia intermedia); 18\% (36) de 21 a 30 años de experiencia (experiencia avanzada), y un 9\% (18) con más de 31 años de servicio (decanos). Del total de los participantes el 51\% (100) son género femenino.

\subsection{Clasificación de CDD}

Para clasificar los resultados de la encuesta en los niveles de desempeño de las CDD (Figura 1) se alinearon los niveles planteados por NETS-T de ISTE (2008), que van de 
principiante a transformador, con los niveles del modelo de Krumsvik (2009), los cuales van desde la adopción hasta la Innovación), cuyas características se presentan en la Tabla 3.

Niveles de desempeño /

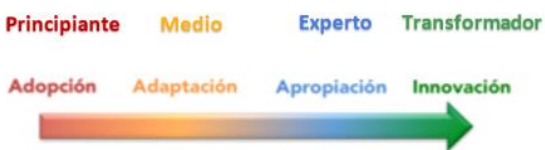

NETS-T de ISTE

Modelo Krumsvik

Figura 1. Niveles de desempeño NETS-T - Krumsvik

Fuente: elaboración propia a partir de ISTE (2008) y Krumsvik (2009; 2014).

Tabla 3. Niveles de desempeño CDD

\begin{tabular}{cl}
\hline Nivel & \multicolumn{1}{c}{ Descriptores de desempeño } \\
\hline $\begin{array}{c}\text { Principiante - } \\
\text { adopción }\end{array}$ & $\begin{array}{l}\text { Profesores que cursan programas de formación docentes que se están iniciando en el uso } \\
\text { de las TIC para mejorar los procesos de enseñanza-aprendizaje. } \\
\text { Uso elemental de las TIC para el ocio fuera del trabajo escolar, y uso básico del software } \\
\text { de aplicación, y herramientas técnicas para la enseñanza en las escuelas. }\end{array}$ \\
\hline $\begin{array}{c}\text { Medio - } \\
\text { adaptación }\end{array}$ & $\begin{array}{l}\text { Docentes que están adquiriendo más experiencia y flexibilidad en la utilización de las } \\
\text { TIC dentro de los ambientes educativos. Además del uso de las TIC para el ocio y la } \\
\text { comunicación, demuestra un uso más eficaz de software de ofimática y herramientas } \\
\text { técnicas para la enseñanza en las escuelas. }\end{array}$ \\
\hline $\begin{array}{c}\text { Experto - } \\
\text { Apropiación }\end{array}$ & $\begin{array}{l}\text { Utiliza las tecnologías digitales de forma eficiente para mejorar los resultados } \\
\text { académicos de los estudiantes, su acción docente y la calidad del centro educativo. Uso } \\
\text { pedagógico reflexivo y perfecta integración de las TIC en disciplinas temáticas, mediante } \\
\text { los sistemas de gestión del aprendizaje. }\end{array}$ \\
\hline Transformador - & $\begin{array}{l}\text { Docentes que exploran, adaptan y aplican las TIC de forma que cambian los procesos de } \\
\text { enseñanza y aprendizaje atendiendo a las necesidades de una sociedad global y digital. }\end{array}$ \\
& $\begin{array}{l}\text { Desarrollo de estrategias de aprendizaje, construcción de conocimiento y metacognición } \\
\text { de las ideas por parte del alumno, formas de evaluación, educación adaptada y sistemas } \\
\text { de gestión del conocimiento. }\end{array}$ \\
\hline & Fuente: elaboración propia a partir de ISTE (2008); Krumsvik (2014).
\end{tabular}

En la Tabla 4 se presenta la clasificación del nivel de CDD en cada dimensión del NETSTT de ISTE (2008), la media sobre la variable general de las CDD, así como la frecuencia de cada nivel de CDD en porcentaje.

Tabla 4. Media y frecuencia del nivel de las dimensiones de las CDD en EMS

\begin{tabular}{cccccc}
\hline $\begin{array}{c}\text { Dimensión } \\
\text { de la CDD }\end{array}$ & Media & $\begin{array}{c}\text { Principiante } \\
\text { Adopción }\end{array}$ & $\begin{array}{c}\text { Medio } \\
\text { Adaptación }\end{array}$ & $\begin{array}{c}\text { Experto } \\
\text { Apropiación }\end{array}$ & $\begin{array}{c}\text { Transformador } \\
\text { Innovador }\end{array}$ \\
\hline I & 3.79 & $1.50 \%$ & $24.40 \%$ & $50.80 \%$ & $23.40 \%$ \\
\hline II & 3.88 & $1.50 \%$ & $21.80 \%$ & $53.30 \%$ & $23.40 \%$ \\
\hline III & 3.79 & $4.10 \%$ & $24.40 \%$ & $44.70 \%$ & $26.90 \%$ \\
\hline IV & 3.73 & $3.60 \%$ & $28.40 \%$ & $45.20 \%$ & $22.80 \%$ \\
\hline V & 3.66 & $4.60 \%$ & $26.90 \%$ & $45.70 \%$ & $22.80 \%$ \\
\hline CDD total & 3.77 & $3.00 \%$ & 25.20\% & $47.90 \%$ & $23.80 \%$ \\
\hline \multicolumn{5}{c}{ Fuente: elaboración propia. } \\
\end{tabular}


Con base en los resultados anteriores, en términos generales la mayoría de los participantes se auto perciben en un nivel de Experto-Apropiación en un 47.90\% (94), seguido de un nivel Medio-Adaptación con un 25.20\% (50), con un 23.80\% (47); contra sólo un 3.0\% (6) que se consideran en un nivel Principiante-Adopción. La puntuación de la media es de 3.77 (sobre 5).

En la clasificación de autopercepción de nivel de CDD se obtuvieron los siguientes resultados: el 49.2\% (97) se auto percibieron en nivel Experto-Apropiación, seguidos por el nivel Medio-Adaptación 29.9\% (59), pero entre estos dos niveles hay 38 casos de diferencia, mismos que coinciden con el número de docentes que se perciben en el nivel Transformador-Innovación. Atendiendo a las consideraciones que hace Krumsvik (2009) sobre el tercer nivel, al referir que el principal desafío de los docentes de hoy, es ante todo, con respecto a la fase de apropiación y el desarrollo de la competencia didáctica de las TIC.

\subsection{Pruebas de asociación}

A efecto de analizar la asociación entre el nivel de autopercepción de CDD y las variables sociodemográficas se utilizó la prueba de Chi-Cuadrado (asociación) y U de Mann-Whitney (comparación), obteniendo los siguientes resultados:

- Edad: se realizó la prueba de Chi-cuadrado, el resultado sugiere que existe una asociación estadísticamente significativa entre la edad del docente y el nivel de CDD $\left(\chi^{2}=27.406\right.$ y su Sig. Asintótica $\left.=.026\right)$; con una fuerza de asociación moderada, de acuerdo con el estadístico V de Cramer (.215).

- Experiencia docente: se realizó la prueba de Chi-cuadrado, el resultado sugiere que existe una asociación estadísticamente significativa entre la experiencia docente en años y el nivel de CDD $\left(\chi^{2}=22.118\right.$ y su Sig. Asintótica $\left.=.036\right)$; con una fuerza de asociación débil, de acuerdo al estadístico V de Cramer (.264).

- Nivel de estudios: prueba de Chi-cuadrado los resultados estadísticos sugieren que no existe una asociación estadísticamente significativa entre el nivel máximo de estudios y los niveles de desempeño de CDD $\left(\chi^{2}=7.372\right.$ y su Sig. Asintótica $=.288$ ).

- Género: se aplicó la prueba de U de Mann-Whitney para muestras independientes, arrojando como resultado que las cinco dimensiones tienen un resultado de $p<.05$, por lo tanto, el resultado de la prueba sugiere: existen diferencias significativas entre el nivel de la CDD y el género.

En resumen, en relación con el análisis de asociación, los resultados estadísticos sugieren que existe una asociación entre las $\mathrm{CDD}$ autopercibidas con las variables edad y 
experiencia docente (medida en años de servicio); sin asociación con el grado máximo de estudios. En cuanto a la variable género el análisis arroja que existen diferencias significativas a razón del género.

\section{Conclusiones}

En la actualidad las competencias digitales para los docentes suponen una ampliación de aprendizajes, formación y recodificación de significados, sobre todo en el nuevo contexto de la sociedad de la información, a "reaprender", no sólo para poder moverse en un mundo digitalizado que demanda nuevas competencias, sino que, además, necesitan dominarlas para su uso pedagógico, lo que multiplica el grado de dificultad al que se enfrentan. Así, los resultados obtenidos sobre el análisis de la asociación entre el nivel de CDD y variables sociodemográficas: edad, experiencia docente y nivel máximo de estudios con base en la evidencia obtenida se concluye que los participantes poseen en lo general una autopercepción positiva acerca de su propio nivel CDD, esto en virtud de que 94 participantes $(47.9 \%$ de la muestra) se autoperciben en un nivel Experto-Apropiación, docentes que según la teoría revisada (ISTE, 2008; Krumsvik, 2009; 2014; Esteve-Mon, 2015; Pérez Escoda, 2016), sugieren que la competencia didáctica de las TIC respecto al uso pedagógico reflexivo y perfecta integración de las TIC en las asignaturas utilizan un aceptable grado de dominio las TIC, diseñando y a veces administrando cursos y evaluaciones en línea, foros de discusión, chat, entre otros. Asimismo, la evidencia obtenida sugiere que a menor edad (hasta 43 años) mayor nivel de CDD; a menor antigüedad o años de experiencia docente mayor nivel de CDD. Por otra parte, atendiendo el análisis comparativo con la variable sociodemográfica género, la evidencia sugiere que existen diferencias significativas entre el nivel autopercibido de la CDD y el género.

\section{Referencias}

DO L394, D. O. (2006). Recomendación del Parlamento Europeo y del Consejo de 18 de diciembre de 2006. Obtenido de http://eur-lex.europa.eu/legalcontent/ES/TXT/PDF/?uri=CELEX:32006H0962\&from=ES

DOF. (2008). ACUERDO número 447 por el que se establecen las competencias docentes para quienes impartan educación media superior en la modalidad escolarizada. Recuperado el 30 de 05 de 2017, de http://dof.gob.mx/nota_detalle.php?codigo=5066425\&fecha=29/10/2008

Esteve-Mon, F. (2015). La competencia digital docente. Análisis de la autopercepción y evaluación del desempeño de los estudiantes universitarios de educación por medio de un entorno $3 d$ (Tesis doctoral). Tarragona, España: Recuperada de: http://www.tdx.cat/handle/10803/291441.

Ferrari, A. (2012). Digital Competence in Practice: An Analysis of Frameworks. European Union. 
Asociación entre el nivel de competencias digitales docentes y variables sociodemográficas en docentes de educación media superior

Gimeno Sacristán, J. (2008). Educar por competencias, ¿qué hay de nuevo? Madrid: Morata. Obtenido de http://galeon.com/chanram/parte1.pdf

Hernández. (2008). La formación del profesorado para la integración de las TIC en el currículum: nuevos roles, competencias y espacios de formación. En A. García-Valcárcel, Investigación y tecnologías de la información y comunicación al servicio de la innovación educativa (págs. 3356). Obtenido de https://dialnet.unirioja.es/servlet/articulo?codigo=2876449

Hernández, L., Acevedo, J., Martínez, C., \& Cruz, B. (2014). El uso de las TIC en el aula: un análisis en términos de efectividad y eficacia. Oaxaca, México: Obtenido de: www.oei.es/historico/congreso2014/memoriactei/523.pdf.

ISTE. (2008). Estándares Nacionales (EEUU) de Tecnologías de Información y Comunicación (TIC) para docentes (2008) NETS-T Por su sigla en Inglés. Obtenido de http://www.iste.org/docs/pdfs/nets-for-teachers-2008_spanish.pdf?sfvrsn=2

Koehler, M. J., \& Mishra, P. (2008). Handbook of technological pedagogical content knowledge (TPCK) for educators. New York: Routledge. Obtenido de https://www.punyamishra.com/wpcontent/uploads/2013/08/TPACK-handbookchapter-2013.pdf

Krumsvik. (2014). Teacher educators' digital competence. Scandinavian Journal of Educational Research, 269-280.

Krumsvik, R. (2009). Situated learning in the network society and the digitised school. European Journal of Teacher Education 32 (2), 167-185. doi:10.1080/02619760802457224

Marquès, P. (2008). Las competencias digitales de los docentes. Obtenido de $\mathrm{http}: / /$ peremarques.pangea.org/competenciasdigitales.htm

Marquès, P. (2012). Impacto de las TIC en la educación: funciones y limitaciones. 3ciencias, 1-15. Obtenido de https://www.3ciencias.com/wp-content/uploads/2013/01/impacto-de-las-tic.pdf

OCDE. (2005). La definición y selección de competencias clave. Resumen ejecutivo. . Obtenido de Organización para la Cooperación y el Desarrollo Económico. : https://www.deseco.ch/bfs/deseco/en/index/03/02.parsys.78532.downloadList.94248.DownloadFi le.tmp/2005.dscexecutivesummary.sp.pdf

Oviedo, P., Cardenas, F., Rendon, M., Zapata, P., Rojas, Y., \& Figueroa, L. (2010). Estilos de enseñanza y estilos de aprendizaje: implicaciones para la educación por ciclos. Revista Actualidades Pedagógicas, (55), 31-43. Obtenido de https://revistas.lasalle.edu.co/index.php/ap/article/view/873

Pérez Escoda, A. (2015). Alfabetización digital y competencias digitales en el marco de la evaluación educativa: Estudio en docentes y alumnos de Educación Primaria en Castilla y León (Tesis doctoral). Salamanca, España: Recuperada de: https://gredos.usal.es/jspui/handle/10366/128252.

Perrenoud, P. (2010). Diez nuevas competencias para enseñar. México: Graó.

Rangel Baca, A. (2015). Competencias docentes digitales: propuesta de un perfil. Pixel-Bit. Revista de Medios y Educación, (46), 235-248. Obtenido de https://recyt.fecyt.es/index.php/pixel/article/view/61622 